\title{
Voigt Fitting is of Special Interest for Impedance and Raman Spectroscopic Analysis for Two-Layered Aurivillius Compound
}

\author{
Md. Abdul Basheer ${ }^{1}$, G. Prasad ${ }^{2}$, and N.V. Prasad ${ }^{3 *}$ \\ ${ }^{1,2,3}$ Material Research Laboratory, Department of Physics, Osmania University, Hyderabad 500007, India \\ ${ }^{*}$ Corresponding Author: nvp1969@ rediffmail.com
}

Available online at: www.isroset.org

Received: 03/Mar/2020, Accepted: 06/Apr/2020, Online: 30/Apr/2020

\begin{abstract}
Bi}_{3} \mathrm{TiNbO}_{9}$ compound is a good ferroelectric with high Curie temperature among other bismuth layered structure ferroelectric (BLSF) of Aurivillius family. To explore the plausible fundamental information about dielectric relaxation, observed in the radio frequency region, a criterion, based on the spectroscopic peak value along with its full width at half maximum, is necessary. In view of this, Voigt profile is fitted to the unsymmetrical bell shaped impedance spectroscopic data of our earlier work made on samarium modified $\mathrm{Bi}_{3} \mathrm{TiNbO}_{9}$ ceramics. In the present investigation, systematic studies like Lorentzian and Gaussian fitting were also made. Voigt profile $\left(V_{f}\right.$ and $\left.V_{f}^{\prime}\right)$ and its parameters are found to be useful to understand the dielectric relaxation mechanism. The results were corroborated to the Raman spectroscopic plots. Such systematic studies are hitherto not reported.
\end{abstract}

Keywords - BLSF, Impedance, Raman spectroscopy, relaxation, Gaussian, Lorentzian, Voigt fitting.

\section{INTRODUCTION}

The Voigt function is considered to be a special and important in depiction of the symmetric feature of any respond peak profile because of its theoretical importance and practically well-fit in to experimental data [1]. It is a known fact that the impedance spectroscopic plots show relaxation peaks in the radio frequency region, correspond to the different relaxing species that are present in the composition. Generally, the dielectric relaxation peaks give information about the competitive interaction of both longrange and short-range ordering that are present in the sample. The same information can be extracted from the specified peak shape and its width [1]. Spectroscopic peaks are generally described either by Gaussian or Lorentzian fitting [2-3]. The former explains about the single relaxation and the later describes multiple relaxation phenomena. In addition, it provides information about the instrument- limitation. The Voigt function $(\mathrm{V}(\mathrm{x}))$ is defined as the convolution between Lorentzian $(\mathrm{L}(\mathrm{x}))$ and Gaussian function $(\mathrm{G}(\mathrm{x}))$, expressed as:

$$
\mathrm{V}(\mathrm{x})=\mathrm{G}(\mathrm{x}) \oplus \mathrm{L}(\mathrm{x})
$$

The Voigt function can interpret the data in twofold: (i) graphical point of view, (ii) numerical point of view. Ida et al [4] pointed out that the Gaussian width and the parameter (a) give an asymptotic explanation for dielectric relaxation data. Here, the parameter (a) defined as the ratio of Lorentzian and Gaussian full width at half maxima (FWHM). Many researchers adopted Voigt fitting to resolve the overlapping line shapes of different modes of Raman spectra [5-7]. Based on this, one can say that the true line-intensity and shape of any spectroscopic data along the instrument distortion can be studied by means of Lorentzian and Gaussian functions. This type of combined analysis in the literature, especially on BLSF is very limited.

In our earlier results, the modulus spectroscopic plots, (complimentary to the dielectric data) have shown broad peaks $[8,9]$. To interpret the relaxation results, alone temperature-dependent dielectric study cannot give information about thermally activated process and therefore a systematic study is necessary. In view of aforementioned, importance, $\mathrm{G}(\mathrm{x}), \mathrm{L}(\mathrm{x})$ and Voigt equation are used to fit the modulus spectroscopy data.

Modulus data can be extracted from the dielectric data. The real and imaginary part of modulus ( $\mathrm{M}^{\prime}$ and $\mathrm{M}^{\prime \prime}$ ) is calculated based on the following equations [10]:

$$
\begin{aligned}
& M^{\prime}=\frac{\varepsilon^{\prime}}{\left(\varepsilon^{\prime 2}+\varepsilon^{\prime \prime 2}\right)} \\
& \mathrm{M}^{\prime \prime}=\frac{\varepsilon^{\prime \prime}}{\left(\varepsilon^{\prime 2}+\varepsilon^{\prime \prime 2}\right)}
\end{aligned}
$$

Where $\varepsilon^{\prime}$ and $\varepsilon^{\prime \prime}$ are the real and imaginary parts of dielectric constant. Here it should be noted that the complex modulus respond function is described as the combination of real and imaginary part of modulus $\left(M^{*}(\omega)\right.$ $\left.=M^{\prime}+i M^{\prime \prime}\right)$.

Gaussian, Lorentz and Voigt equations are given in the following expressions: 


$$
\begin{aligned}
\mathrm{Y}=\mathrm{Y}_{0}+\left(\frac{\mathrm{A}}{\mathrm{w} \times \sqrt{\frac{\pi}{2}}}\right) \\
\quad \times \exp \left(\frac{-2\left(\left(\mathrm{x}-\mathrm{x}_{\mathrm{c}}\right)\right.}{\mathrm{W}}\right)^{2}(\text { Gaussian })(4)
\end{aligned}
$$$$
\mathrm{Y}=\mathrm{Y}_{0}+\left(2 \times \frac{\mathrm{A}}{\pi}\right) \times\left(\frac{\mathrm{w}}{\left(4 \times\left(\mathrm{x}-\mathrm{x}_{\mathrm{C}}\right)^{2}+\mathrm{W}^{2}\right)}\right) \quad(\text { Lorentz) }(5) \mathrm{Y}=
$$$$
\mathrm{Y}_{0}+\left(\frac{\mathrm{A} \times 2 \times \ln (2) \times \mathrm{w}_{\mathrm{L}}}{\pi^{1.5} \times \mathrm{w}_{\mathrm{G}}{ }^{2}}\right) \times \int\left(\frac{\exp \left(-\mathrm{t}^{2}\right)}{\left(\sqrt{\ln (2)} \times \frac{\mathrm{wL}_{\mathrm{L}}}{\mathrm{w}_{\mathrm{G}}}\right)^{2}}\right)+(\sqrt{4 \times \ln (2)} \times
$$$$
\left.\frac{\left(\mathrm{x}-\mathrm{x}_{\mathrm{c}}\right)}{\mathrm{w}_{\mathrm{G}}-\mathrm{t}}\right)^{2} \text { (Voigt) }
$$

Here $\mathrm{Y}_{0}$ is the offset value; $\mathrm{A}$ is area under the curve and $\mathrm{W}$ is width of the curve and $\mathrm{X}_{\mathrm{c}}$ center of peak position. The Gaussian and Lorentzian parameters, $\mathrm{w}_{\mathrm{G}}$ and $\mathrm{w}_{\mathrm{L}}$ are related to FWHM of Gaussian and Lorentzian [5, 11].The peak area, width and peak position were also obtained by fitting the Voigt function, using the equation (6).

The main purpose of the present study is to extract the following inheriting properties:

i). Explanation about change over mechanism (long-range to short-range order vice versa).

ii) The plausible relaxation mechanism can be understood by means of parameters, obtained by fitting the data with $\mathrm{L}(\mathrm{x}), \mathrm{G}(\mathrm{x})$ and Voigt equations, in the lower frequency domain of imaginary part of modulus spectroscopic data.

iii) Finally, the results are corroborated to Raman spectroscopic fitting data.

\section{II . METHODOLOGY}

$\mathrm{Bi}_{3-\mathrm{x}} \mathrm{Sm}_{\mathrm{x}} \mathrm{TiNbO}_{9}(\mathrm{BSTN}-\mathrm{x}, \mathrm{x}=0.20 .40 .60 .8 \& 1.0)$, single phase ceramics were prepared by solid state route. The reactant oxide powders were AR grade with $99.99 \%$ purity. The powders of $\mathrm{Bi}_{2} \mathrm{O}_{3}, \mathrm{Sm}_{2} \mathrm{O}_{3}, \mathrm{TiO}_{2}$ and $\mathrm{Nb}_{2} \mathrm{O}_{5}$ were taken in a stoichiometric ratio. The powders were mixed thoroughly using agate mortar and pestle. The dried mixture was pre-sintered at $900^{\circ} \mathrm{C}$ for 2 hour. Requisite amount of polyvinyl alcohol was added as a binder before making it into pellets. The pressed pellets were once again sintered at $1050-1100^{\circ} \mathrm{C}$ for $5 \mathrm{~h}$. A detailed synthesis process was described elsewhere [9]. The impedance measurements were made by Hewlett-Packard (HP4192A) impedance analyser. Raman spectroscopic data is obtained by using Nd-YAG-532 nm LASER alpha 300 of WitecGermany made.

\section{RESULTS AND DISCUSSION}

To understand the defect role, the imaginary part of modulus with frequency at $450^{\circ} \mathrm{C}$ is shown in Figure 1, for all the samples. The data is fitted up to specified peak in lower frequency range only. Dotted, dash and scattered lines represents a Lorentzian, Gaussian and Voigt fitting. As seen in the parameters obtained (not shown) from the fitting is incomplete and may not give any conclusions and therefore an alternative profile is necessary.
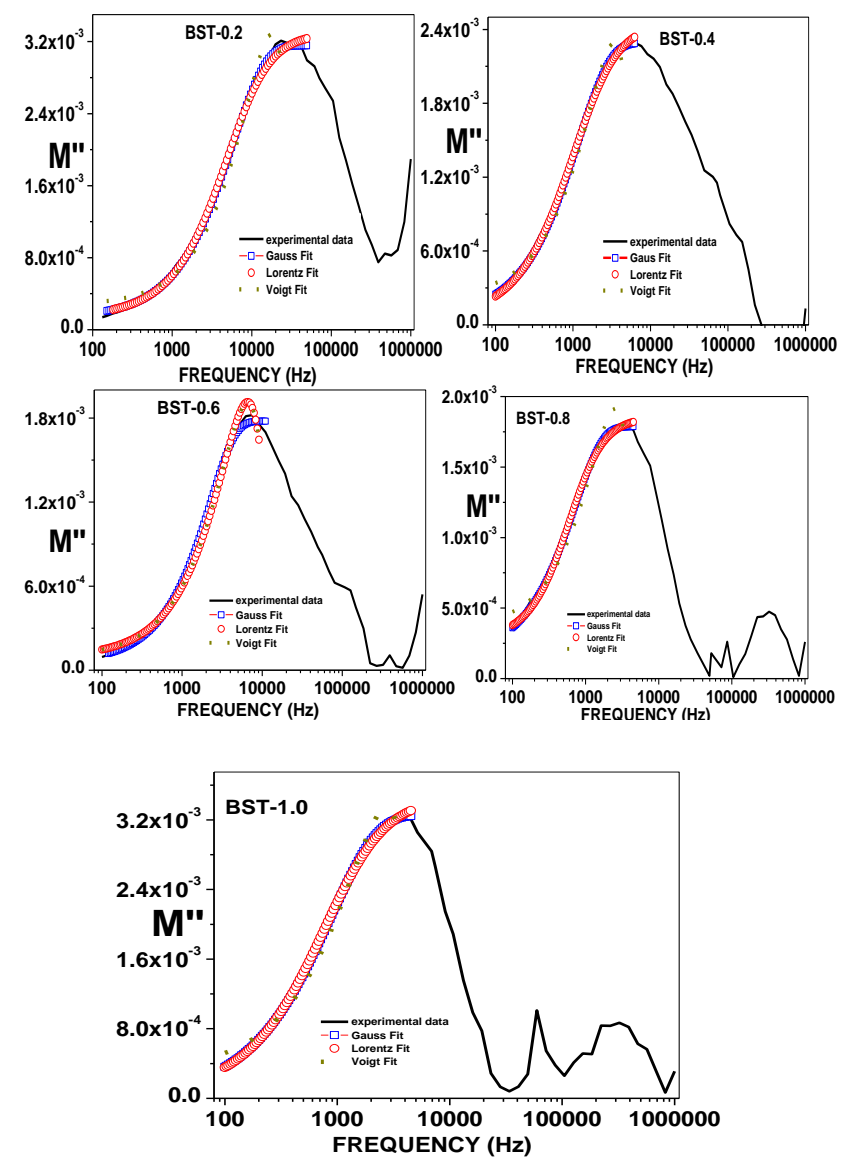

Figure 1. Gaussian, Lorentzian and Voigt fitting for BSTN-x

$(x=0.20 .40 .60 .81 .0)$

Raman spectroscopy is well known technique to identify the chemical species and its vibrational modes. Figure 2 shows the Raman spectra for all the samples of BSTN-x.
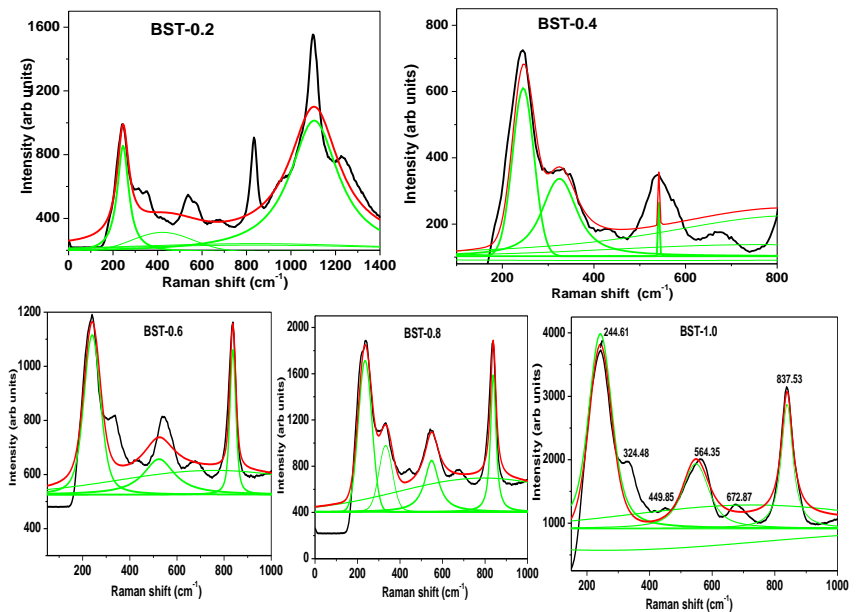

Figure 2. Raman shift Vs. Intensity (arb. units) with respect to BSTN-x Samples. 
It is important to resolve the broad Raman peaks and its vibrational modes by means of convolution of Lorentzian $\left(\mathrm{L}_{\mathrm{w}}\right)$ and Gaussian $\left(\mathrm{G}_{\mathrm{w}}\right)$ functions. Therefore one can accurately understand the line width and peak position of Raman spectra by fitting the spectra using Voigt function. However in literature many researchers were quoted [1113], that Pseudo-Voiget profile $\mathrm{V}_{\mathrm{w}}(\mathrm{x})$ gives the explanation for specific modes which are inherently hidden the function $\mathrm{V}_{\mathrm{w}}(\mathrm{x})$, expressed as:

$$
\mathrm{V}_{\mathrm{w}}=\eta \mathrm{L}_{\mathrm{w}}+(1-\eta) \mathrm{G}_{\mathrm{w}}
$$

Where Lorentzian (Lw) and Gaussian (Gw) profiles are described as

$$
\begin{gathered}
L_{w}=\frac{\gamma \pi^{-1}}{(w-c)^{2}+\gamma^{2}} \\
G_{w}=\frac{1}{\sqrt{2 \pi} \gamma} \exp \frac{-(w-c)^{2}}{2 \gamma^{2}} \\
L_{w}=\frac{\gamma \pi^{-1}}{(w-c)^{2}+\gamma^{2}}
\end{gathered}
$$

Voigt profile is calculated by adopting the following two methods.

(1) The complete Voigt profile is calculated by using the equation :

$$
\boldsymbol{f}_{v}=\rho \times W_{G}+\rho \times W_{L}
$$

Where $\rho$ is arbitrary value (in the present case by assuming as: $\rho=0.3,0.7$ and 1.0$)$

$W_{G}, W_{L}$ are related (proportional) to FWHM of Gaussian $\left(\Gamma_{\mathbf{G}}\right)$ and Lorentzian $\left(\Gamma_{\mathbf{L}}\right)$ respectively.

(2) Voigt profile can also be constructed by considering height time width or height divided by width of the peaks.

From the equation (8), the Voigt function profile can be approximated from the experimental value, therefore the following equation is more evident and suitable in the present study. Based on the arbitrary values of $\rho$ and FWHM of Gaussian $\left(\hat{\Gamma}_{\mathbf{G}}\right)$ and Lorentzian $\left(\hat{\Gamma}_{\mathrm{L}}\right)$ Voigt profile function $\left(f_{v}\right)$ is approximated and finally given in the following equation $[12,13]$.

$$
\mathrm{f}_{\mathrm{V}}\left(\mathrm{x} ; \hat{\Gamma}_{\mathrm{G}}, \dot{\Gamma}_{\mathrm{L}}\right)=\left[\frac{1}{\hat{\Gamma}_{\mathrm{G}}+\dot{\Gamma}_{\mathrm{L}}}\right] \times \rho \times\left[\frac{\mathrm{x}}{\hat{\Gamma}_{\mathrm{G}}+\dot{\Gamma}_{\mathrm{L}}}\right]
$$

Figure 3 shows the variation of Voigt profile as a function of arbitrary values of $\mathrm{x}$ for chosen different arbitrary $\rho$ values (say $\rho=0.3,0.7 \& 1.0$.). The variation of Voigt function with different arbitrary values of $x$ gives the complete picture of Voigt profile. By using the above equation, all $\mathrm{f}_{\mathrm{v}}$ values were calculated for all samples. From the Figure.3, it is observed that $\mathrm{f}_{\mathrm{v}}$ is found to increase with increasing the arbitrary parameter $\rho$ and $\mathrm{x}$. The $\mathrm{f}^{\prime}{ }_{\mathrm{v}}$ (slope values obtained from the Figure.3) were also found to increase with the arbitrary $(x)$ values. The kind of theoretical Voigt profile can be visualized by giving different $\rho$ and $\mathrm{f}_{\mathrm{v}}$ values. It should be noted here that ideal condition, $\mathrm{W}_{\mathrm{G}}+\mathrm{W}_{\mathrm{L}}=1$ is maintained while evaluating the $f_{v}$.
Figure.4 (a) shows the variation of $\mathrm{f}^{\prime}{ }_{\mathrm{v}}$ with the composition. Many researchers are pointed out that Gaussian width is more important when compared to Lorentzian; however the ratio between Gaussian and Lorentzian is always less than 1.Several asymptotic expressions are necessary to analysis the data. In this connection, Voigt profile is seems to be more valid. In the Voigt profile ' $a$ ' and ' $b$ ' defined as:

$$
a=\frac{W_{L}}{W_{G}} \& b=\frac{x}{W_{G}}
$$

Where $w_{L}$ and $w_{G}$ are related to $\Gamma_{\mathrm{L}}$ and $\Gamma_{\mathrm{G}}$.

Based on the above equation a, value is calculated for all the samples and shown in inset Figure.4 (a)
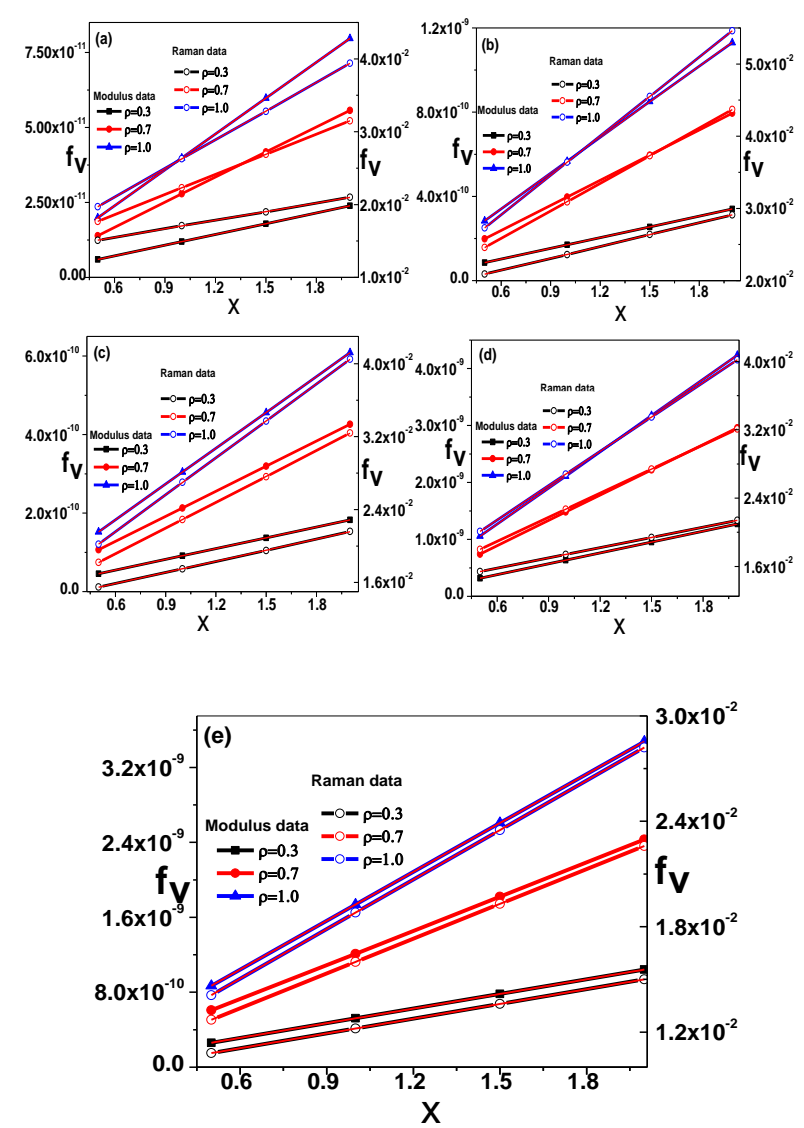

Figure 3. Voigt Profile of Modulus and Raman data as a function of augment at different ${ }^{\rho}$ values 


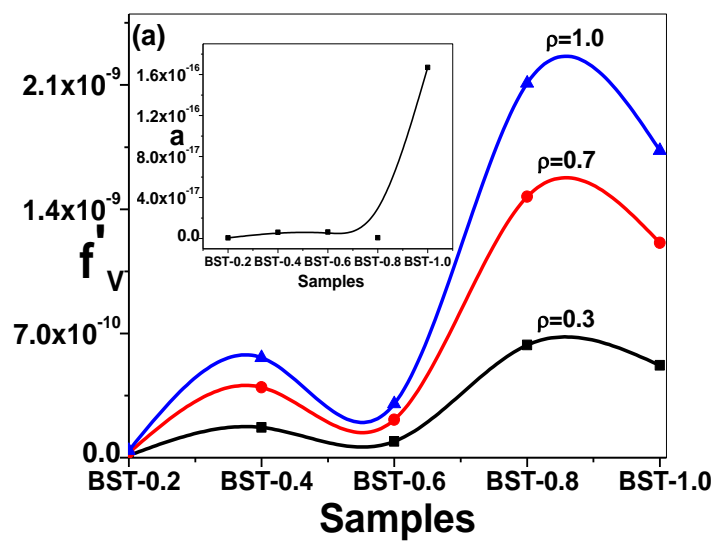

Figure $4(a) . f^{\prime}{ }_{v} \quad V s$. BSTN-x (obtained from modulus data) Inset Figure .Parameter (a) Vs. sample composition (SBTN-x)

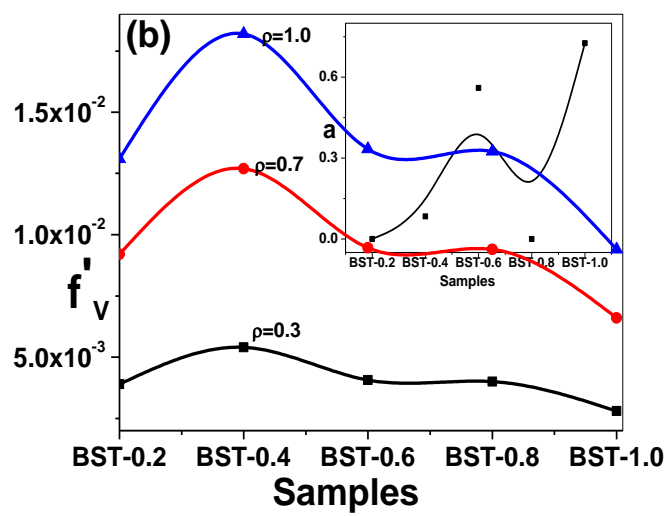

Figure $4(b) . f^{\prime}{ }_{v} V s$. BSTN-x samples (obtained from Raman data) Inset Figure. Parameter (a) Vs sample (BSTN-x)

In order to analyse the Raman spectrum (shown in Figure.2) one has to resolve the overlapping line shapes. The line width and peak positions were taken from fitting the Raman spectroscopic plot (Figure.2).Figure.3 also shows, the Voigt profile obtained for different composition of Raman spectra of BSTN spectroscopic data (shown as marked open circles in Figure.3). From the figure 3 the variation of $\mathrm{f}$ ' $\mathrm{v}$ with the sample composition is shown in Figure.4 (b).The parameter, calculated by the adopting similar approach made in earlier impedance results. The variation of ' $a$ ' with respect to all the samples is shown as inset figure.4 $(b)$.

It has been reported in literature that the oxygen vacancies, which are created due to the fact of bismuth loss at higher temperature. These vacancies get accumulated at the grain boundaries and acts as opposite polarizing process and hence they may result into broad peaks near $450^{\circ} \mathrm{C}$ in all the samples [9]. Moreover the multiple rare-earth ionic incorporation at bismuth site creates more vacancies or complex defect dipoles owing to fact of electron hopping process in a single ionized on doubly ionized vacancies (Kroger-Vink reaction). This kind of hopping mechanism is also established in single crystals, grown by gel technique. Recent report on mixed Cobalt Levo-Tartrate crystals grown by gel technology have shown limited hopping mechanism and the results are reflected in their dielectric data [14].

The following Kroger-Vink model suggests that increase in the temperature reduces the disorder and creates electron-captured oxygen vacancies, according to equations:

$$
\begin{aligned}
& {\left[\mathrm{NbO}_{5} \cdot \mathrm{V}_{O}^{x}\right]+\left[\mathrm{NbO}_{6}\right]^{x} \rightarrow\left[\mathrm{NbO}_{5} \cdot \mathrm{V}_{O}^{\prime}\right]+\left[\mathrm{NbO}_{6}\right]^{\prime}} \\
& {\left[\mathrm{NbO}_{5} \cdot \mathrm{V}_{O}^{\prime}\right]+\left[\mathrm{NbO}_{6}\right]^{x} \rightarrow\left[\mathrm{NbO}_{5} \cdot \mathrm{V}_{O}^{\prime \prime}\right]+\left[\mathrm{NbO}_{6}\right]^{\prime}} \\
& {\left[\mathrm{NbO}_{5} \cdot \mathrm{V}_{O}\right]+\frac{1}{2} \mathrm{O}_{2} \rightarrow\left[\mathrm{NbO}_{6}\right]}
\end{aligned}
$$

Where $\left[\mathrm{NbO}_{6}\right]$ ' is donor, $\left[\mathrm{NbO}_{5} . \mathrm{V}_{\mathrm{O}}{ }^{\prime}\right]$ is donor-acceptor and $\left[\mathrm{NbO}_{5} . \mathrm{V}_{\mathrm{O}}{ }^{\prime \prime}\right]$ is acceptor vacancies. Here, the oxygen vacancies from complexes defect dipoles in the intermediate structure. In this structure, $\left[\mathrm{NbO}_{5} \cdot \mathrm{V}_{\mathrm{O}}{ }^{\mathrm{x}}\right]$ complex cluster is a donor candidate and $\left[\mathrm{NbO}_{6}\right]^{\mathrm{x}}$ is an acceptor candidate.

The results are consistant with the degree of preferred orientation $\left(\mathrm{P}_{\mathrm{c}}\right)$ and $\mathrm{A}$-site ionic radii with composition [9] shown in figure 5 . The same can be seen in 'a' parameter value, as shown in inset figure $4(a-b)$. The relative error estimated in the present Voiget profile is found to be very less (below 1\%). The relative error profile fitting (E), calculated by using the following formula:

$$
\mathrm{E}=\frac{\sum_{i}\left|\left(\frac{y_{i}}{y_{\max }}\right)-b-I\left(x, \hat{\Gamma}_{\mathrm{G}}, \hat{\Gamma}_{\mathrm{L}}\right)\right|}{\sum y_{i_{\max }}}
$$

$\mathrm{Y}$ is the ratio of intensity to maximum intensity value $y i / y_{\max }$, the term $b$ represents background value . The value of $b=1$ for maximum background and $b=0$ for having no back ground, and $\mathrm{x}$ is the arbitrary closer value and $\Gamma_{\mathrm{G}}$ and $\Gamma_{\mathrm{L}}$ are its used significances.

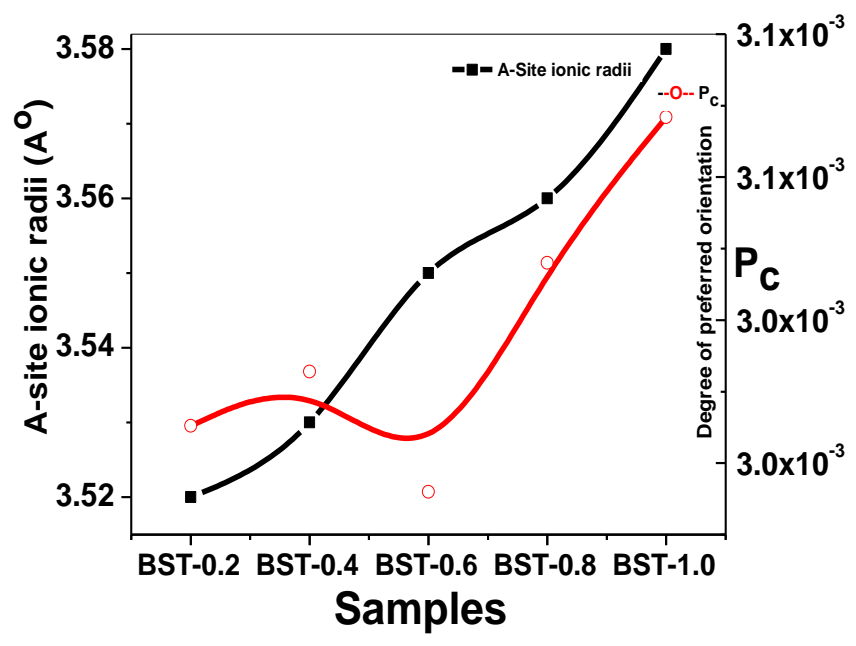

Figure 5 .A-site Ionic radii and degree of preferred orientation Vs. all the samples $(B S T N-x)$ 


\section{CONCLUSION AND Future SCOPE}

The convolution function of both Gaussian and Lorentzian gives the most important description of symmetric feature of the peak. The theoretical interpretation gives the complete picture of the experimental data keeping the instrumental limitations. Since the single impedance spectroscopic approach may not be sufficient to explain the asymmetric or non-Debye behavior, therefore, the complete picture of relaxation requires an advanced mathematical approach is necessary. It has also reported that pseudo-Voigt approximation is best fitting among all other fittings. All the spectroscopic Raman curves of experimental data were well fitted by Voigt profile. The true vibrational modes can be approximated by convolution of Gaussian $\mathrm{G}(\mathrm{x})$ and Lorentzian $\mathrm{L}(\mathrm{x})$ functions. The standard deviation observed in these functions is found to be within the experimental error. The proposed Voiget function and its profile are clearly comparable to impedance and Raman spectroscopic results. The error found in profile is typically less than $1 \%$ even considering large background value $(b=1)$. A conclusion is arrived that discrimination of the continuous (broad) peak and the function represents augment, which depends on Voigt profile. However, further studies are needed to speculate the complete Voigt profile.

\section{REFERENCES}

[1] J. I Lang ford, "Proceedings of International Conference on Accuracy in powder diffraction II", National institute standards and technology, USA, pp.110-126, 1992.

[2] Th. H De Keijser , J.I Langford, E.J Mittemeijer and A.B.P Vogels," Use of Voigt function in a single line method for the analysis of X-ray diffraction line broadening ", J.Appl.Cryst, Vol.15,pp.308-314, 1982.

[3] H.M Rietveld, "A profile refinement method for nuclear and magnetic structures", J.Appl.Cryst, Vol.2, pp.65-71, 1969.

[4] T Ida, M Ando and H Toraya , "Extended pseudo-Voigt function for approximating the Voigt profile" ,Vol. 33,pp.1311-1316, 2000.

[5] H.O Rocco, D.I, Iriarte and J Pomarico, "General expression for the Voigt function that is of special interest for applied spectroscopy”, Appl.Spectroscopy,Vol.55,pp.822-826,2001.

[6] J.D Weatherston, N.C Worstell and H.J Wu, "Quantitative surface-enhanced Raman spectroscopy for kinetic analysis of aldol condensation using Ag-Au core-shell nanotubes", The Analyst, 2016.

[7] T Sundius, "Computer fitting of Voigt profiles to Raman lines", Journal of Raman Spectroscopy, Vol.1, issue.5, pp.471-488, 1973.
[8] V.J UshaPraveena, N.V Prasad, G Prasad and G.S Kumar, "Electrical studies on double rare earth modified $\mathrm{Bi}_{6} \mathrm{Fe}_{2} \mathrm{Ti}_{3} \mathrm{O}_{18}$ ", Vol.514, issue .1, pp.61-69, 2017.

[9] M.D AbdulBasheer, N.V Prasad, G Prasad and G.S Kumar, "Dielectric studies onSm-modified two layered BLSF ceramics", Bull.Mater.Science, Vol.42,pp.1776-6,2019.

[10]. J Ross Macdonald, "Impedance Spectroscopy”, John Wiley and Sons, New York, 1987.

[11] G.K Wertheim, M.A Butler , K.W West and D.N.E Buchana, "Determination of the Gaussian and Lorentzian content of experimental line shapes. Review of scientific Instruments", Vol.45, issue.11,pp.1369-1371, 1974.

[12] S Alstrom Tommy, N Schmidt Mikkel, Tomas Rindzevicius, Anja Boisena, "pseudo -Voigt component model for highresolution recovery of constituent spectra and raman spectroscopy" IEEE international conference on Acoustics, speech and signal processing (ICASSP), New Orieans, LA, pp.2317-2321, 2017.

[13] N.H. Manani, H.O. Jethva and M.J .Joshi, "Dielectric Relaxation, Conductivity Mechanism and Complex Impedance Spectroscopic Studies of Pure and Cadmium Mixed Cobalt Levo-Tartrate Crystals", Int.J.SRPAS, Vol.o8, issue.1,pp.08-15,2020.

[14] G.P.M Poppe and C.M.J Wijers , "More efficient computation of the complex error function.ACM Transactions on mathematical software $(T O M S),, .16: \quad$ pp 38-46, 1990.

\section{AUTHORS PROFILE}

Md. Abdul Basheer passed MSc, from Osmania University in the year 2004. He is currently pursuing Ph.D. in the Department of Physics, Osmania University, and currently working as Assistant Professor in Physics, Deccan College of Engineering and Technology, Nampally, Hyderabad, Since 2011.

Prof. G. Prasad has completed his M.Sc (Physics) with Electronics specialization from Osmania University. He has done his Doctorate in solid state physics from Indian Institute of Technology, Kharagpur. He has worked as Lecturer in Physics, St. Jospeh college, Bangalore and Lecturer in Materials Science, Mangalore university before joining Osmania University in 1989. His research interests include finite element analysis for material modelling and impedance analysis studies of ferroelectric materials. He has guided 10 Ph.D students and published 150 research papers. He has also authored four books.

Dr. N. V. Prasad obtained his Ph.D from Osmania University in the year 2001. He has more than twenty years of academic and research experience, presently working as a Assistant Professor. in Department of Physics, Osmania University, Hyderabad. His research interest includes ferroelectric and magnetoelectric materials. He has more than 55 international journals. He is a life member of Indian Science Congress Association, Kolkatta. 